\title{
LA LETTERATURA INSEGUE LA SCIENZA. UNA PROSPETTIVA SETTECENTESCA
}

\author{
FRANCO ARATO \\ (University of the Witwatersrand)
}

\begin{abstract}
The aim of the paper is to study the relationship between literature and science from the point of view of Eighteenth century writers and philosophes who, having received a scientific education, tried to explain the sense of the Galileian and Newtonian world in layman's language: the struggle for clarity - often nurtured by imagination - was part of the battle for the triumph of Enlightenment. The article analyzes Fontenelle's Entretiens sur la pluralité des mondes (a seminal work but still influenced by Cartesian cosmology), the Italian translation of Lucretius by Alessandro Marchetti, inspired by the atomism of Gassendi's physics, finally the early vulgarizations of Newton. Special emphasis is put on Voltaire's Élémens de la philosophie de Newton and on Algarotti's Newton for the Use of the Ladies, two very different but equally successful books. In the age of Enlightenment literature and science seemed to engage with the same problem: to read without prejudice the 'Book of the World' (to cite a famous expression by Galilei) and to defend the libertas philosophandi, the freedom to investigate.
\end{abstract}

Almeno in due modi la letteratura può interrogare la scienza. Esiste lo scrittore che alla scienza, scegliendo di ignorarne le finalità, chiede suggestioni, immagini, paradossi, anticipazioni del futuro; e c'è il caso di letterati che, avendo avuto una formazione scientifica, si propongono la sfida di spiegare quelle stesse scienze al laós, cioè al pubblico dei non intendenti, con parole vere (o almeno verosimili) ma comuni. Si danno naturalmente molti casi intermedi, che possono rendere problematica questa partizione: per esempio, il 
celebre Gödel, Escher, Bach di Douglas R. Hofstadter (1979) è un libro di fantascienza o di divulgazione? Gli scienziati ne trovano noiose le pagine scritte à la Lewis Carroll, i laici confessano di non capire quasi niente del cosiddetto teorema dell'incompletezza lì a lungo analizzato. $\mathrm{Ci}$ sono anche autori che condivisero, senza apparenti interferenze, due mestieri: per esempio, Geoffrey Chaucer, il grande emulo inglese di Boccaccio, redasse a fine Trecento un fondamentale Trattato sull'astrolabio che i lettori dei Canterbury Tales possono ignorare senza loro danno. Se si prende per buona, in via transitoria, la distinzione tra science fiction e divulgazione scientifica, bisogna comprendere nella prima schiera, per esempio, Cyrano de Bergerac (États et empires de la lune et du soleil) e Jules Verne, Clive Staples Lewis (Perelandra) e Fredric Brown, Isaac Asimov e cento altri nostri contemporanei; nella seconda, su cui principalmente mi soffermerò, per esempio Bernard Le Bovier de Fontenelle ${ }^{1}$. Dal quale comincio, perché con lui

1 In queste pagine faccio riferimento, nell'ordine, alle seguenti edizioni (nella trascrizione di tutti i testi ho conservato la grafia originale). Bernard Le Bovier de Fontenelle, Entretiens sur la pluralité des mondes, Paris, Blageart, 1686, seconda stampa «augmentée d'un nouvel entretien», ivi, Guerout, 1687. Per il volgarizzamento di Lucrezio da parte di Marchetti, la cui rara princeps è londinese G. Pickard, 1717, disponiamo di due edizioni moderne: a cura di M. Saccenti, Modena, Mucchi 1992, e a cura di D. Aricò, Roma, Salerno, 2003. Cito la versione inglese di Thomas Creech del De Rerum Natura (1682) dalla terza edizione, Titus Lucretius Carus, his Six Books of Epicurean Philosophy done into English Verse, with Notes, London, Sawbridge, 1683 (ho consultato la copia che è nella Cullen Library, University of the Witwatersrand). L'Antilucretius sive de Deo et natura libri novem. Opus posthumum [...] del cardinale di Polignac è citato dall'edizione di Parigi del 1747; la traduzione italiana, Anti-Lucrezio di F.M. Ricci, è veronese, Carattoni, 1751. La Petite cosmogonie portative di Raymond Queneau (Paris, Gallimard, 1950) è stata tradotta da Sergio Solmi, con prefazione di Italo Calvino, Torino, Einaudi, 1982. Gli Élémens de la philosophie de Newton di Voltaire (1738) si leggono nell'edizione critica della Voltaire Foundation (London-Geneva, 1992), a cura di R.L. Walters e W.H. Barber. Il Newtonianismo per le dame di Francesco Algarotti (Milano, 1737, con la falsa indicazione di Napoli) si legge nella versione definitiva (intitolata Dialoghi sopra l'ottica neutoniana, 1764) nelle Opere di F. Algarotti e S. Bettinelli, a cura di E. Bonora, Milano-Napoli, Ricciardi, 1969; recentissima una nuova edizione commentata delle Poesie di Algarotti, a cura di A.M. Salvadè, Milano, Aragno, 2009. Cito il Poème sur le desastre de Lisbonne e il Poème sur la loi naturelle 
siamo nel bel mezzo della cosiddetta rivoluzione scientifica e nel gran secolo delle contese letterarie (la famosa querelle des anciens et des modernes). Con Fontenelle la Natura fu generosa: accordandogli non solo un ingegno non comune, ma anche un tempo straordinariamente lungo per coltivarlo. Nato a Rouen nel 1657 e morto a Parigi nel 1757, appartiene al club ristretto, allora più che ora, dei centenari. Un aneddoto vuole che Madame de Grimaud, una nobildonna che sarebbe morta a centotré anni, incontrando una volta il quasi coetaneo Fontenelle gli sussurrasse, tra la costernazione e lo stupore: «La Mort nous a oubliés», avendo questa risposta dal valente vegliardo: «Chut! Elle pourrait nous entendre». Vero o non vero, l'aneddoto registra la passione per il bon mot che fu propria dell'epoca (sarebbe meglio dire: delle epoche) in cui Fontenelle visse. Eccone almeno un altro suo in rima, che vien bene per i pregiudizi di tutte le conventicole di sapienti: «Personne a de l'esprit, / sauf nous et nos amis» (nessuno è intelligente, salvo noi e i nostri amici). Quest'uomo molto spiritoso pare si fregiasse però del serio motto di Spinoza, secondo cui il filosofo è tenuto non a ridere o a piangere delle cose della vita, ma a capire (intelligere). Il libro che gli diede celebrità è giovanile, scritto prima dei trent'anni, gli Entretiens sur la pluralité des mondes (apparsi anonimi nel 1686). Una ripetizione del romanzo

di Voltaire nell'edizione di Ginevra, Cramer, 1756. Per le opere di Antonio Conti: Prose e poesie, due tomi, Venezia, Pasquali, 1739-1756. Sui rapporti tra letteratura e scienza la bibliografia è imponente; mi limito a segnalare, per il secolo che qui interessa: Scienza e letteratura nella cultura italiana del Settecento, a cura di R. Cremante e W. Tega, Bologna, Il Mulino, 1984; Fernand Hallyn, Les structures rhétoriques de la science de Kepler à Maxwell, Paris, Seuil, 2004; Men, Women and the Birthing of Modern Science, edited by J. Zinsser, De Kalb, Northern Illinois University Press, 2005; Simone Mazauric, Fontenelle et l'invention de l'histoire des sciences à l'aube des Lumières, Paris, Fayard, 2007. Sull'età successiva si veda il recente libro di Richard Holmes, The Age of Wonder. How the Romantic Generation discovered the Beauty and Terror of Science, London, Harper, 2008. 
fantascientifico di Cyrano? Non proprio. In quel libro di libera conversazione c'è un po' di science fiction ma c'è ancor più la scienza contemporanea, cioè la cosmologia di Cartesio, su cui Fontenelle giurò sempre, anche quando nel continente europeo si diffusero le idee di Newton. Figlio di un influente avvocato che sedeva nel parlamento di Normandia, Fontenelle fu alunno nel collegio dei gesuiti della sua città, ricevendone tra l'altro un'ottima formazione matematica: pubblicò (tardi, nel 1727) degli Éléments de la géométrie de l'infini che hanno un piccolo posto nella storia del calcolo infinitesimale. Fu per oltre mezzo secolo Segretario dell'Académie des Sciences e, spiace dirlo, un po' responsabile della resistenza tutta francese alla penetrazione, in Accademia e altrove, delle idee newtoniane. Scrisse per il teatro (non a caso era nipote di Pierre e di Thomas Corneille), compose poesie, opere di critica e di filosofia, trattati d'ogni tipo, tra i quali è da segnalare un'Histoire des oracles che è una pungente critica, in parte tratta dal libro del medico olandese Anthony Van Dale, del modo prescientifico di guardare al mondo e, insieme, una sottile analisi dei residui di paganesimo vivi tra i cristiani: «l'affaire des oracles», spiegava Fontenelle, «estoit de sa nature une affaire de Religion chez les Payens», dunque «en est devenuë une sans necessité chez le Chrestiens». Precisazione un po' ironica: che infatti meritò una lunga replica del padre Jean François Baltus, della Compagnia di Gesù. L'ironia non manca neanche negli Entretiens sui mondi, ben funzionale, in questo caso, all'ispirazione socratica dell'insegnamento impartito: destinato ancora a pochi, non alla vasta platea cui guarderanno i divulgatori dell'età romantica.

Le sei conversazioni serali tra lo scrittore e una marchesa, précieuse sì, ma quasi mai ridicule, sono recitate in idillica cornice: un bel parco in una fresca estate, sotto un magnifico cielo stellato 
contro cui meglio risaltano i biondi capelli della dama. Sin dalla prefazione Fontenelle si premura di precisare il senso del titolo di fronte alle eventuali obiezioni di «gens scrupuleux, qui pourront s'imaginer qu'il y a du danger par rapport à la Religion» (anche per aver scritto de l'infinito universo, e mondi - vecchia sentenza epicurea! - Giordano Bruno, meno di un secolo prima, era stato condannato). Fontenelle si trae elegantemente d'impaccio: poiché le Scritture ci insegnano che tutti gli uomini discendono da Adamo, e non risulta che Adamo abbia colonizzato lo spazio, «j’y [nei mondi] mets des habitans qui ne sont point du tout des hommes. Que sont-ils donc? Je ne les ay point veus, ce n'est pas pour les avoir veus que j'en parle». Qui è accennato un 'partito' epistemologico molto caro a Fontenelle, motore di tutta l'operetta: bisogna l'avere sempre «l'esprit curieux» e «les yeux mauvais», perché, dice alla marchesa il filosofo degli Entretiens, se voi aveste occhi migliori «vous verriez bien si les étoiles sont des soleils qui éclairent autant des mondes, ou si elles n'en sont pas», e dunque non metterebbe neanche conto parlarne.

Un paradosso? Un'eresia antibaconiana e antigalileiana, visto che il nostro Linceo solo rendendo più acuti i suoi occhi riuscì a vedere i famosi «quattro pianeti intorno alla stella di Giove» (e molto altro)? Non è così: Fontenelle vuol dirci che non dobbiamo sempre fidarci dei nostri sensi, schiavi del senso comune, e che anche la scienza (lui la chiama «philosophie» ma sta parlando d'astronomia) procede per congetture e per sforzi di immaginazione. Un po' di sforzo d'immaginazione è per esempio necessario per far capire alla marchesa (nel primo dialogo) che la tesi copernicana è vera (e bella), falsa (e sgraziata) la teoria tolemaica. Immaginazione, non arbitrio: il mondo pensato da Fontenelle è sì «bien méchanique»e, nel paragone, «la Nature est 
un grand Spectacle qui ressemble à celuy de l'Opera», dove c'è un «machiniste caché dans le parterre»; però il filosofo ammonisce la marchesa a non cedere al «faux merveilleux» di chi vorrebbe farci credere che la Natura sia «une espèce de magie». Erano passati ben cinquantaquattro anni dai Massimi sistemi di Galileo (1632), ma i devoti, almeno in Italia, continuavano a scrivere in testa ai loro libri, si trattasse d'opere di scienza o di poesia, che la tesi eliocentrica (o «pitagorica»), non ancora dimostrata, era avanzata a titolo di «pura ipotesi matematica». Non tutti al mondo erano egualmente conformisti di fronte ai decreti del Sant'Uffizio: e infatti nell'edizione parigina del 1686 e nella ristampa del 1687 la corriva formula manca. Ma in Francia, si sa, c'era un re geloso delle sue prerogative. Naturalmente gli Entretiens finiro subito all'Indice (22 settembre 1687). Gli argomenti accennati nel primo dialogo per spiegare il moto terrestre non sono dissimili da quelli già usati da Galileo nella sua opera di divulgazione. Riassumiamoli. La terra sembra immobile ma «c'est la mesme chose - spiega lo scrittore francese alla sua interlocutrice - que si vous vous endormiez dans un bateau qui allast sur la rivière»; al mattino vi svegliereste sulla stessa barca «dans la mesme place, et dans la mesme situation à l'égard de toutes les parties du bateau». Nuove però sarebbero le rive: cioè, fuor di metafora, le cosiddette stelle fisse che appaiono all'osservatore: «je suis sur la terre et la terre décrit un grand cercle autour du soleil. Je regarde au centre de ce cercle, j'y voys le soleil. S'il n'effaçoit point les étoiles en poussant ma vue en ligne droite au-delà du soleil, je le verrois nécessairement repondre à quelques étoiles fixes, mais je voys aisément pendant la nuit à quelles étoiles a repondu le jour», e poiché la terra cambia posto, è neccessario ch'io veda al mattino il sole «répondre» a altre stelle; dunque «c'est-là le rivage qui change tous les jours». Del resto, come già 
aveva spiegato Giordano Bruno, il pianeta terra apparirebbe simile alla nostra luna agli ipotetici osservatori lunari: «cette moitié de la lune qui a la nuit, estant tournée vers la moitié de la terre qui a le jour, nous voit sans estre vue, et nous voit sous la mesme figure que nous voyons la pleine-lune. C'est alors pour les gens de la lune pleine-terre, s'il s'est permis de parler ainsi». Per Fontenelle, che segue la spiegazione meccanicistica cartesiana, i pianeti si muovono «au milieu de la matière celeste qui est mille plus fluide que l'eau» come una barca si muove lungo la corrente di un fiume.

La luna è abitata? È l'argomento del secondo dialogo, in parte ispirato a un vecchio libro del vescovo anglicano John Wilkins, The Discovery of a World in the Moon, or a Discourse tending to prove that it's probable there may be another habitable World in that Planet (1638). Fontenelle pensa di poter azzardare quell'ipotesi, sostanzialmente negata da Galileo, ma non cessa tuttavia, dice, «de vivre civilement avec ceux qui ne le croyent pas», dicendosi pronto ad allinearsi «à leur opinion avec honneur, si elle avoit le dessus». Fa un sorridente paragone: un borghese di Parigi, dalle torri di Notre-Dame non distinguendo abitanti nella vicina Saint-Denis, potrebbe dedurne, erroneamente, che quella città è deserta. Ma questa è una «sottise», replica la marchesa, perché tutti, scorgendo almeno i tetti di Saint-Denis, dovrebbero capire che quella città è tal quale Parigi. Ma anche la luna, replica il filosofo, non è difforme dalla terra: ha i suoi monti, le sue valli, le sue pianure, come già aveva spiegato Ariosto, autore molto amato in Francia e a lungo citato negli Entretiens. Deduzione approssimativa e incauta, lo sappiamo, ma Fontenelle da buon letterato è pronto a moralizzarci sopra: «Nous voulons juger de nous, nous en somme trop près; nous voulons juger des autres, nous en sommes trop loin». E un gran guaio però voler a tutti costi antropomorifizzare la realtà celeste; 
c'è l'esempio pronto, quasi a compensare l'azzardo lunare, delle bizzarre teorie avanzate nei secoli per spiegare le eclissi prima delle scoperte cinque-secentesche. Ancor oggi nelle Indie orientali «on croit que quand le soleil et la lune s'éclipsent, c'est qu'un certain Démon qui a les griffes fort noires, les étend sur ces astres dont il veut se saisir»; meno indulgente di quanto siamo noi oggi di fronte alle offerte dell'illusionismo etnico, lo scrittore francese con sarcasmo già illuministico precisa: «vous voyez pendant ces tempslà les rivières couvertes de testes d'indiens qui se sont mis dans l'eau jusqu'au cou, parce que c'est une situation très devote selon eux, et très propre à obtenir du soleil et de la lune qu'il se défendent bien contre le Démon». Il mondo antico è istruttivo e curioso, anche nei suoi errori, il moderno è però quasi sempre più sagace: nei Nouveaux dialogues des morts, altra operetta fontenelliana, precedente gli Entretiens, c'è per esempio una conversazione nei Campi Elisi tra il medico ellenistico Eresistrato e il moderno William Harvey, lo scopritore della circolazione del sangue (De motu cordis et sanguinis in animalibus, 1628). A cosa serve questa gran scoperta, chiede indispettito il vecchio anatomista greco; risponde impassibile l'inglese: «On n'a peut-estre pas encore eu le loisir de tirer quelque usage de tout ce qu'on a appris depuis peu, mais il est impossible qu'avec le temps, on n'en voye de grands effets». Alle domande della scienza il letterato risponde con un razionale atto di fede intorno all'idea che l'accrescimento del sapere teorico possa rivelarsi comunque utile in un prossimo futuro. Nel caso specifico, possiamo dargli torto?

I restanti tre dialoghi fontenelliani si soffermano sugli ipotetici abitanti di Mercurio, Marte, Giove e Saturno, non su quelli del Sole, ovviamente: «il $\mathrm{n}$ y a pas moyen d'y mettre d'habitans. Le pourquoy non? nous manque là». Un sesto dialogo, aggiunto nella 
seconda edizione, dà ragguaglio delle recenti scoperte realizzate coi telescopi, in particolare dell'avvistamento di un ciclone di color rosso che circonda l'atmosfera di Giove. Un posto d'onore ha nel libro la teoria cartesiana, erronea ma allora prevalente, dei «tourbillons», i vortici: escogitati dal grande Renato anche per giustificare (come s'esprime la dubbiosa marchesa di Fontenelle) il fatto che ci siano «des planètes qui tournent autour d'autres planètes qui ne valent pas mieux qu'elles»; non sarebbe più ragionevole e più uniforme, almanacca la dama, che «toutes les planètes, grandes et petites, n'eussent que le mesme mouvement autour du Soleil»? L'idea dei vortici, «terrible» ma anche «agréable», sembra fatta apposta per soddisfare l'immaginazione del pubblico, con quel poco, o quel tanto, di sospensione emotiva ch'essa porta con sé: abbiamo forse da temere che un pianeta, trasportato dal fluido celeste, ci trascini prima o poi dentro un suo vortice? E poi i movimenti delle comete possono turbare l'ordine cosmico? Fontenelle col solito gusto del capovolgimento dei luoghi comuni così rassicura l'interlocutrice: «nous voyons nous autres estre bien malheureux quand il nous paroist une comète; c'est la comète ellemesme qui est bien malheureuse». Eppure l'universo non è immutabile, come avevano creduto Aristotele e gli aristotelici: «les anciens ont vû dans le Ciel des étoiles fixes que nous n'y voyons plus. Ces soleils ont perdu leur lumière». Emerge il tema, sempre perturbante in ogni epoca e sotto ogni cielo, della temporalità, ovvero della storicità del cosmo. Non c'è da stupirsi che la marchesa, con femminile sensibilité, si immalinconisca e anche un po' si ribelli di fronte a quest'idea per lei (e per molti lettori d'allora) nuova. Perché ci è impossibile comprendere intuitivamente questo aspetto della vita della Natura? Perché gli 
antichi, così sapienti, non ne ebbero una percezione netta (per quanto forse, dico io, l'idea di ekpúrosis degli stoici vi si avvicinò)?

Qui Fontenelle introduce, con eleganza d'artista, una sorprendente comparazione: se le rose di un giardino, che durano un solo giorno, potessero scrivere «des histoires»e «des mémoires», e le lasciassero in eredità alle successive quindicimila generazioni di rose, scriverebbero forse del loro giardiniere sempre la stessa cosa: «Nous avons toujours vû le mesme jardinier, de mémoire de rose on n'a vû que luy, il a toujours esté fait comme il est assurément, il ne meurt point comme nous; il ne change seulement pas. Le raisonnement des roses - conclude sornione il filosofo - seroit-il bon?». Le rose sono naturalmente gli uomini, il giardiniere rappresenta i fenomeni del mondo (dei mondi). In questi mondi il creatore non è, come ovvio, negato, ma rimane davvero «caché», nascosto, come aveva scritto il pio e profondo Pascal. C'è forse un «machiniste caché dans le parterre», ma non ci è dato vederlo, né intuire le sue intenzioni. Tra galanteria e filosofia, tra entusiasmo per le conquiste della scienza e malinconia per l'inesorabile scansione delle leggi del cosmo, Fontenelle fornì all'Europa un modello letterario destinato a intrattenere generazioni di lettori. Il Fontenelle del pieno Settecento, il grande, più grande, Voltaire, nato trent'anni dopo, si esercitò, pur ammirando l'esprit del predecessore, a demolire la forma letteraria degli Entretiens, non meno che la sostanza scientifica, diventando l'alfiere delle idee di Newton: il quale, bisogna ricordarlo, pubblicò i suoi Principia mathematica, in cui la cosmologia cartesiana è confutata ed è enunciata la teoria dell'attrazione universale, nel 1687, proprio l'anno dopo la stampa degli Entretiens. Strano il destino dei letterati che si innamorano delle scienze. In chiusura del libro Fontenelle aveva regalato alla sua impaziente marchesa, ansiosa di saper tutto, 
un altro dei suoi famosi bons mots: i veri scienziati sono come gli elefanti, «qui en marchant ne posent jamais le second pied à terre, que le premier n'y soit bien affermy». Ma stavolta un elefante inglese aveva superato il mercuriale bello spirito francese...

Prima di parlare del Voltaire newtoniano è bene fare una sosta tra i cultori e i produttori di versi nell'Italia di fine Seicento. Dove la dispersione della scuola galileiana, conseguente la famosa condanna, non aveva impedito lo sviluppo di alcuni settori specifici degli studi: la trigonometria con Bonaventura Cavalieri (15981647); la meccanica dei gas e dei fluidi con Evangelista Torricelli (1608-1647); la fisiologia e l'astronomia con Giovanni Alfonso Borelli (1608-1679). Quest'ultimo, che era stato allievo di Benedetto Castelli (il primo scolaro di Galilei), è forse il più importante tra gli scienziati italiani di secondo Seicento, benché non molto noto fuori dalla cerchia degli specialisti: a lui si deve la fondazione della iatromeccanica in Italia, nonché l'approfondimento della meccanica di Keplero (secondo Koyré, Borelli fu addirittura a un passo dall'intuire $i$ princìpi dell'attrazione universale newtoniana). A Pisa Borelli ebbe fra $\mathrm{i}$ suoi allievi il toscano Alessandro Marchetti (1633-1714): fisico, astronomo, medico - scienziato non di prima grandezza, è il caso di dirlo -, fu influenzato dal neo-atomismo che proveniva dalla vicina Francia; più felicemente Marchetti fu poeta, soprattutto traduttore degli antichi: Anacreonte, Virgilio, Lucrezio (in Arcadia entrò subito, nel 1691, col nome di Alterio Eleo). La traduzione in endecasillabi sciolti del lucreziano De rerum natura, opera giovanile (1664-1669 circa), è al centro di uno dei più curiosi casi di censura letteraria di quell'epoca, che coinvolse scienziati, uomini di Stato e di Chiesa. Diffuso in centinaia di manoscritti in Italia e fuori, il Lucrezio toscano (che già Leibniz cita con entusiasmo nella 
Théodicée, 1710) dovette attendere l'anno 1717 per essere stampato: in edizione postuma e londinese, a cura di un poeta e librettista allora soggiornante in Inghilterra, Paolo Rolli. La proibizione romana fu sollecita e senz'appello (16 novembre 1718): proibizione che naturalmente fulminava non la qualità poetica, che piacque anche a monsignori e cardinali, ma il contenuto filosofico dell'originale, reso finalmente accessibile a tutti. Rolli pensò bene di dedicare il poema al principe Eugenio di Savoia: grande uomo d'armi, eroe cristiano al servizio dell'Impero nella guerra contro il Turco («Prinz Eugenius der edle Ritter...», cantavano i suoi soldati), ma anche raffinato conoscitore d'arte e di poesia, in odore di deismo se non di libertinismo. Per controbilanciare la presenza di tal nome, lo stesso Rolli aveva messo in evidenza la prolissa, troppo marziale protesta del defunto autore, che aborriva (sono parole scritte da Marchetti nel lontano 1668) gli «empi suoi [di Epicuro] dogmi intorno all'anima umana ed al sommo Iddio», e aggiungeva d'essere «prontissimo (ogni qualvolta il bisogno ciò richiedesse) non solo ad impiegare tutto l'ingegno e le forze mie, ma anco a spargere tutto il mio sangue, avvegnaché io mi pregi veramente d'esser filosofo, ma più mi glorii d'esser cristiano». In altre, più tarde edizioni compaiono in testa al Della natura delle cose versi scritti dallo stesso Marchetti per Luigi XIV, re di Francia (cui aveva inizialmente pensato di dedicare la versione). Sono versi? Sembra piuttosto una giaculatoria: «O dell'Eterno Padre, o dell'Eterno / Figlio, Eterno, ineffabile, infinito, / vicendevole Amor, Amor fecondo, / Santo Amor, vero Amor, unico Amore, / unico Amor, che da principio il Cielo / creasti, e l'aureo Sol cinto di raggi...» (e via così per altre tre pagine). Ancora più tardi, nel 1779, il fiorentino Gioacchino Cambiagi ripubblicò Marchetti di nuovo a Londra, con una sonante dedica, in prosa, al còrso Pasquale Paoli 
esule in Inghilterra, uomo di cui era ammiratore e storico: nella prefazione a questa lussuosa e assai corretta ristampa (che riceverà le lodi del Carducci) Cambiagi non mancava di deplorare il «bigottismo» del granduca Cosimo III, che un secolo prima aveva proibito la traduzione.

L'attività del Marchetti poeta è strettamente legata alla scienza contemporanea; anzi il suo primo pensiero, spiega in una letteraconfessione ad Antonio Magliabechi del luglio 1675, era stato quello di «spiegare $[\ldots]$ in un poema i segreti della natura» al fine non solo d'«essere annoverato tra $\mathrm{i}$ buoni poeti», ma anche di assolvere il proprio «debito come professore di filosofia e di matematica». Per farsi la mano Marchetti aveva tradotto i versi di un antico; al grande Gian Domenico Cassini, l'astronomo ligure che fu al servizio del re Sole, quasi vent'anni dopo (dicembre 1694) aveva però ribadito la sua intenzione di spiegare, dice, «in versi sciolti toscani quella filosofia che, non obbligata ad autore alcuno né moderno né antico, a me pare più sensata e ragionevole». Progetto sfumato: Marchetti non scrisse mai un poema didascalico moderno (lo scriverà un po' dopo, come vedremo, un illustre cardinale francese), e dobbiamo allora accontentarci, si fa per dire, della sua meravigliosa traduzione lucreziana. Eccone almeno il fastoso inizio (che rende l'«Aeneadum genetrix, hominum divomque voluptas» e quel che segue):

Alma figlia di Giove, inclita madre del gran germe d'Enea, Venere bella, degli uomini piacere e degli Dèi:

tu che sotto i girevoli e lucenti segni del cielo il mar profondo e tutta d'animai d'ogni specie orni la terra, che per sé fòra un vasto orror solingo; 
te, Dea, fuggono i venti; al primo arrivo

tuo svaniscon le nubi; a te germoglia

erbe e fiori il suolo industre;

tu rassereni i giorni foschi, e rendi

col dolce sguardo il mar chiaro e tranquillo,

e splender fai di maggior lume il cielo.

Nella più libera Inghilterra, che tacitamente consentì alla pubblicazione postuma del Lucrezio italiano, sin dal 1682 - può essere interessante ricordarlo - Thomas Creech (1659-1700) aveva reso integralmente per la prima volta in inglese il De rerum natura in heroic couplets rimati. Classicista di formazione oxoniense, traduttore di Teocrito, Orazio, Giovenale, Manilio, a quarantun'anni Creech si suicidò (o per amore, o per debiti, non si sa): in questo, se dobbiamo dar retta alla vita lucreziana di Eusebio, emulando il grande poeta latino (come subito osservarono i cronisti di allora). Ecco, a specchio, l'esordio del Lucrezio di Creech:

Kind Venus, glory of the best abodes,

Parent of Rome, and joy of men and gods;

Delight of all, comfort of sea and earth;

To whose kind powers all creatures owe their birth.

At thy approach, Great Goddes, streight remove

What e're are rough, and enemies to love;

The clouds disperse, the winds do swiftly wast,

And reverently in murmurs breath their last:

The earth with various art (for thy warm powers

That dull mass feels) puts forth their gawdy flowers:

For Thee doth subtle luxury prepare

The choicest stores of earth, of sea, and air. 
Soluzioni energiche ma meno eleganti di quelle del quasi contemporaneo Marchetti: bisogna aspettare Pope e i poeti della sua cerchia - una generazione dopo - perché il classicismo inglese trovi la miglior misura. Tuttavia il libro di Creech, fortunatissimo all'epoca e universalmente ammirato, non manca d'interesse storico, soprattutto per il piccolo, succoso apparato di note: Epicuro vi è certo condannato, ma il suo sistema viene preso sul serio. Si afferma, è vero, che l'atomismo fa a pugni con la «known and demonstrated property of quantity, infinite divisibility»; si ammette tuttavia che i celebri versi lucreziani del primo libro che (ispirandosi all'etica del maestro) stigmatizzano il sacrificio di Ifigenia possano essere letti «with much profit and satisfaction», come quelli di un «excellent satyrist against the heathen worship, for he [Lucrezio] severely scourges the mad zeal of Mensacrificers».

$\mathrm{Ma}$ - legge dell'indivisibilità a parte - quanto era attuale a fine Seicento dal punto di vista scientifico l'atomismo che rivive negli esametri lucreziani? Il ritorno nell'Europa moderna del pensiero di Epicuro, e della poesia del suo vessillifero latino, coincide in gran parte con la diffusione dell'opera di Pierre Gassendi (1592-1655) e con il postulato, contenuto nel De motu gassendiano (1643), di uno spazio illimitato, postulato che è tra l'altro premessa alla rivoluzione della cosmologia newtoniana. Marchetti, come anche Creech, intuì l'importanza del tentativo di Gassendi di superare il meccanicismo cartesiano. L'italiano nel quinto libro del suo Lucrezio (vv. 518 ss.: l'universo e le parti che lo compongono sono mortali) decise di introdurre proprio il nome del moderno Gassendi, novello Epicuro (poi, pentitosi o impauritosi, casserà quel nome):

Questa stessa cagione e questa stessa 
Natura delle cose, ancor che molto sia che già fu trovata, ormai del tutto quasi sepolta in sempiterno oblio, pur di fresco è risorta, vie più vaga e più bella che mai, per le immortali opre del gran Gassendo, onore e lume del bel paese ove la Senna inonda. Et io pur or principalmente, io stesso fui trovato fra tanti, et ebbi in sorte d'esporla altrui nella paterna lingua pria d'ogni altro Toscan come dettolla per entro ai dotti suoi carmi robusti pria d'ogni altro romano il gran Lucrezio.

Tono orgogliosamente profetico (anche se un po' duro nel ritmo, che qui sa di prosa): quasi che Marchetti si sentisse latore d'una verità arcana eppure attualissima.

Sappiamo quanto risulti contagiosa la forza della poesia. Se l'antico Lucrezio appariva così vero, oltre che così bello, nella lingua della letteratura e delle scienze moderne, forse era il caso, pensò qualcuno, di preoccuparsi un po' e di correre ai ripari. Fu l'idea di un grande letterato e diplomatico francese di casa in Italia, il cardinale Melchior de Polignac (1661-1741: una generazione dopo Marchetti), autore degli oltre dodicimila esametri latini dell'Antilucretius sive de Deo et Natura (postumo, 1747). Voltaire, che pure di solito non era tenero con chi vestiva la porpora, nel Temple du goût definì Polignac «oracle de la France». Oltre che letterato finissimo (fu tra gli immortali dell'Académie), il cardinale prestò il suo servizio alla diplomazia di Luigi XIV: fra l'altro negoziò a Utrecht $\mathrm{i}$ trattati che posero fine alla guerra di successione spagnola, l'ultima disastrosa impresa militare del Re 
Sole (1713). La memoria delle sue doti persuasive rivive nelle parole attribuite ad Alessandro VIII (Pietro Ottoboni, certo non uno sprovveduto): «Je ne sais comment vous faites - avrebbe detto il papa al Polignac nel 1690 durante le estenuanti trattative tra Roma e la Francia relative al clero gallicano -, vous paraissez toujours être de mon avis, et c'est moi qui finis par être du vôtre». Evidentemente confidando in queste sue capacità di persuasore, Polignac cominciò a comporre nel 1697 in Olanda - durante una lunga, forzata pausa dai propri incarichi al servizio del re - il poema in perfetti esametri lucreziani in cui si proponeva di convincere un esprit fort, cui dà il nome di Quintus, della verità della religione cristiana e, in subordine, della fisica e della metafisica cartesiane: un po' a specchio di Lucrezio, che aveva dedicato il suo poema a un amico, l'oratore e pretore Caio Memmio, simpatizzante dei circoli epicurei. Chi è il Quinto dell'Antilucretius? Non lo sappiamo. Sappiamo però che l'idea del libro balenò nella mente del francese dopo lunghi, amichevoli colloqui con Pierre Bayle (1647-1706), il grande giornalista, erudito e storico calvinista da molti sospettato di deismo, se non proprio di ateismo. Ricordo fra l'altro che Bayle, curioso di scienza come tutti gli uomini cólti dell'età sua, aveva pubblicato a Rotterdam nel 1682 le Pensées à l'occasion de la comète qui parut au mois de Décembre 1680 in cui dimostrava come l'apparizione delle comete non fosse un fatto imprevedibile, né tanto meno presagio di eventi miracolosi. Polignac fece leggere a molti i suoi esametri e continuò per tutta la vita a limarne la forma $\mathrm{e}$ ad aggiornarne il contenuto: ora dando conto dell'ottica di Newton, che gli pareva degna d'attenzione, benché egli restasse fedele in generale al sistema dei vortici di Cartesio; ora affilando le sue armi contro il temibilissimo John Locke, il sensista inglese che aveva fra l'altro insinuato che la materia forse potesse pensare (orrore!, 
ovvero «tetricus horror», V, 1042). Nei salotti, dove pure Polignac, come tutti gli uomini potenti e affascinanti, aveva anche dei nemici, l'Antilucretius faceva furore, e soprattutto le donne reclamavano, per lor maggiore intelligenza, che i versi fossero tradotti in francese, e in italiano (il cardinale visse a lungo a Roma). Se Polignac non fece in tempo a pubblicare il libro in vita, fu per un suo demone d'incontentabilità, mescolato forse a certa sprezzatura snobistica. Per essere un'opera di divulgazione che voleva dimostrare la compatibilità tra religione e scienza moderna, l'Antilucretius risultava in effetti un prodotto per palati troppo raffinati: Polignac s'era divertito nei suoi nove libri, parzialmente costruiti sulla falsariga dei sei del poeta latino, a praticare un gioco allusivo. Se per esempio al verso 101 del primo libro Lucrezio aveva così commentato il sacrifico della povera Ifigenia, immolata ad Artemide dal padre Agamennone: «tantum religio potuit suadere malorum» (con Marchetti: «error sì grave / persuader la religion poteo»); il cardinale aveva risposto esecrando coloro che mettono il mondo all'arbitrio della Fortuna con l'emistichio «Tantum potuit suadere libido» (I, 18). Polignac era naturalmente prudente quanto spettava a un diplomatico e a un principe della Chiesa: se in cuor suo non dubitava della bontà della teoria eliocentrica di Copernico, nel libro ottavo dell'Antilucretius (vv. 88-149) esponeva in buon ordine, senza prendere partito, $\mathrm{i}$ «tria de toto systemata cognita mundo», il tolemaico, il copernicano appunto, e quello di Tycho Brahe (l'astronomo danese aveva cercato un compromesso: la terra immobile intorno cui ruotano il sole e la luna, tutti gli altri pianeti ruotando a loro volta attorno al sole): salvo osservare che era un po' singolare la volontà dell'uomo d'essere al centro dell'universo («hic Mundi consistere centrum / vult et ait»). Chi aveva orecchi... 
Come tutti i cartesiani, il cardinale paventava l'idea newtoniana di uno spazio assoluto, indipendente da qualunque Motore, temendo che tale teoria potesse portare all'ateismo (era anche l'opinione del suo amico Montesquieu). Newton aveva parlato d'attrazione universale, affermando di non voler fingere ipotesi? Ebbene, gli risponde Polignac, in ciò restaurava senza saperlo la «virtus occulta» degli aristotelici: obiezione non profonda, ripetuta mille volte in Francia e altrove. Ecco i pochi versi in questione nella traduzione, ben presto eseguita (1751) con dedica al cardinale Angelo Maria Querini, dall'abate benedettino romano Francesco Maria Ricci (libro quarto, vv. 1065 ss.):

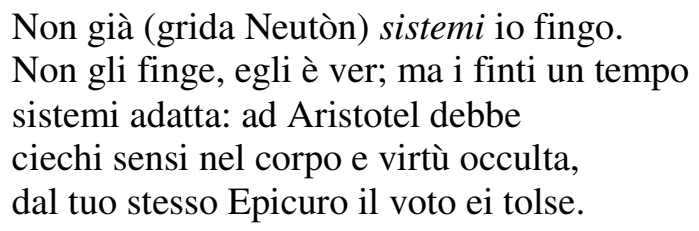

Il 'genio' della poesia scientifica forse non ha mai abbandonato la Francia. Duecento anni dopo l'Antilucretius il filosofo e letterato Raymond Queneau (1903-1976) stupì i suoi molti ammiratori con una Petite cosmogonie portative (1950: poi tradotta in Italia da Sergio Solmi e commentata da Italo Calvino) che racconta in millequattrocento nitidi alessandrini la storia della terra: dal Bigbang («éclatement de l'atome primitif») ai computer («machines à calculer»).

Restiamo nel secolo dei lumi. Contro le obiezioni della scuola cartesiana a Newton, si mosse il già più volte evocato Voltaire. François Marie Arouet (1694-1778), il poeta, romanziere e filosofo a tutti noto, ebbe un'istruzione scientifica appena al di sopra della mediocrità; solo dopo i trent'anni cominciò a interessarsi 
seriamente di fisica. L'occasione fu un lungo soggiorno a Londra (maggio 1726 - novembre 1728), raccontato poi, in termini filosofici e anche aneddotici, nelle Lettres anglaises, ovvero Letters concerning the English nation (1733: la traduzione inglese precedette curiosamente l'originale francese). Avendo trovato superiore al francese il sistema politico inglese, che aveva le sue basi nella tolleranza religiosa (secondo la formula accattivante: «un Anglais comme homme libre va au Ciel par le chemin qui lui plaît»), Voltaire intuì che anche la scienza newtoniana dovesse essere migliore di quella insegnata nelle scuole di casa. Una viva impressione destò in lui il solenne funerale di Newton (20 marzo 1727), cui volle assistere: in quell'occasione scoprì l'alto prestigio sociale di cui godeva la scienza in Inghilterra, e rifletté allora quanto improbabile sarebbe stata una sepoltura di Cartesio (se lo scienziato fosse morto non a Stoccolma ma in patria) a Saint-Denis, accanto alle tombe dei re (Newton fu sepolto a Westminster vicino ai grandi d'Inghilterra). Già nelle Lettres cominciò a divulgare qualche idea sull'ottica e sulla fisica di Newton, lavorando tuttavia su testi di seconda mano. Tornato in Francia, dove nel frattempo s'era innamorato d'una gran donna che di matematica ne sapeva molto più di lui, Émilie de Breteuil marchesa du Châtelet, nel castello di Cirey (nell'Alta Marna) cominciò a studiare Newton sul serio e produsse infine nel 1738 gli Élémens de la philosophie de Newton, che segnano una svolta fondamentale nella conoscenza della nuova fisica sul continente europeo. Per prudenza Voltaire fece uscire la prima edizione in Olanda: ma un po' gli spiacque che lo stampatore di Amsterdam avesse finito per utilizzare nel frontespizio l'esca pubblicitaria «à la portée de tout le monde». Il filosofo amava indubbiamente la popolarità, non la demagogia, visto che sapeva quanto difficile fosse capire Newton. L'epistola di 
dedica a Émilie aveva un incipit provocatorio: «Ce n'est point ici une marquise, ni une philosophie imaginaire». Tutti capirono al volo che Voltaire alludeva a Fontenelle e ai suoi dialoghi galanti di mezzo secolo prima : antiquati ormai, nella forma non meno che nel contenuto; la Châtelet era anch'ella marchesa, ben inteso: non in quanto marchesa, però, il filosofo le si rivolgeva, ma perché autentica femme savante, seguace della vera filosofia e per questo ribattezzata dal suo ammiratore, e amante, «Minerve de France, immortelle Émilie». Del resto Voltaire mostrava d'avere idee chiare sulla destinazione della scienza: «la philosophie est de tout état et de tout sexe, elle est compatible avec la culture des belles-lettres, et même avec ce que l'imagination a de plus brillant».

È notevole che gli Élémens, libro rigoroso e perentorio nella sua didascalica chiarezza, contengano una prima parte metafisica in cui Voltaire espone problemi apparentemente non scientifici: lo spazio e la durata come proprietà di Dio, la libertà del Creatore e quella dell'uomo, la religione naturale e rivelata, la natura dell'anima. È vero che Newton, anche per smentire i suoi detrattori che lo accusavano di irreligione, aveva apposto in fondo ai Principia philosophiae naturalis lo «Scholium generale» dove dichiarava la sua fede in un Dio pantokrátor, onnipotente (Newton era sinceramente cristiano, appartenente alla setta non conformista degli antitrinitari: e lasciò una montagna di scritti intorno alle Scritture e alla cronologia sacra, oltreché di argomento alchemico). Voltaire volle rovesciare l'ordine: prima di parlare dell'ottica, della dinamica e della cosmologia mise in chiaro che «toute la philosophie de Neuton conduit nécessairement à la connaissance d'un Être suprême qui a tout créé, tout arrangé librement»; se la materia gravita, «elle ne gravite pas de sa nature, ainsi qu'elle est étendu de sa nature: elle a donc reçu de Dieu la gravitation» (I, 1). 
Non si trattava, come qualcuno credette, d'una semplice strategia per tener buoni i gesuiti francesi (i quali recensirono per altro con entusiasmo il libro sul «Journal de Trévoux: «tout Paris bégaye Newton, tout Paris étudie et apprend Newton»); erano domande che Voltaire faceva spesso a se stesso (esiste un Essere intelligente onnipotente?) e che avevano trovato provvisoria risposta nei teologi d'ispirazione newtoniana, da lui spesso ascoltati a Londra, in pubblico e in privato: teologi che non gli spiacevano anche in quanto nemici di Leibniz. Voltaire, si sa, non amava i preti, e infatti s'entusiasmò quando in Inghilterra incontrò un quacchero il quale, alla domanda perché mai essi non disponessero di ministri del culto, aveva risposto con un'ironica controdomanda: «pourquoi abandonnerons nous notre enfant à des nourrices mercenaires quand nous avons du lait à lui donner?» (Lettres anglaises, II). Ma forse non è altrettanto noto che Voltaire aveva in sospetto anche gli atei. Il problema del Sommo Bene era per lui inestricabilmente legato con quello del Sommo Male. Come si capisce leggendo il Candide, o ancor meglio i drammatici versi ispirati dal tremendo terremoto di Lisbona (Poème sur le désastre de Lisbonne, 1755): «Élemens, animaux, humains, tout est en guerre. / Il le faut avouer, le mal est sur la terre»; e c'è di peggio: «La Nature est muette, on l'interroge en vain; / on a besoin d'un Dieu qui parle au genre humain». La Natura muta? Parole un po' sorprendenti in bocca all'autore degli Élémens su Newton, al convincente espositore del nuovo sistema del mondo. Ma il terremoto di Lisbona ebbe su Voltaire quasi lo stesso effetto che su Lucrezio aveva avuto la peste di Atene, la catastrofe naturale con cui si chiude, o meglio s'interrompe, il $D e$ rerum natura. La Natura è comprensibile in quanto è, non perché è. A fine secolo altri newtoniani penseranno altro; pare per esempio che l'astronomo Pierre-Simon Laplace abbia così risposto a 
Napoleone, che gli domandava che posto c'era per Dio nella sua Mécanique: «je n'ai pas besoin de cette hypothèse-là».

Il Voltaire divulgatore è chiaro senza mai essere corrivo. Qualche eccesso polemico nei confronti di Descartes (che pure era stato il primo, contemporaneamente a Snell, a definire le leggi della rifrazione) è spiegabile con la volontà di sbaragliare il partito cartesiano, ancora forte alla Sorbona e nell'Académie des sciences. Per altro la Francia accademica pareva ormai pronta a sopportare l'affronto: proprio nei mesi in cui Voltaire scriveva il suo libro, il francese Maupertuis, accompagnato da Clairaut e dallo svedese Celsius, approdava in Lapponia e verificava l'esattezza del calcolo newtoniano della curvatura dell'arco di meridiano sotto il polo. A Parigi, commentò sorridendo, si pensa ancora che la terra abbia la forma di un melone... I geodeti, gente scettica ed eminentemente pratica, avevano confermato il calcolo tutto teorico di Newton, matematico sedentario. Della bellezza e semplicità del sistema newtoniano Voltaire discorre in vari luoghi. Per esempio, rigettando il «pieno» e i vortici cartesiani ed esponendo le leggi della gravitazione e dell'attrazione, osserva: «cette démonstration est d'autant plus belle et plus puissante, qu'elle est indépendante de toute observation, et fondée sur la mécanique primordiale du monde». E là dove indica la scoperta dei sette colori primitivi, si compiace di osservare come già Newton nell'Ottica (I, II, 6, 2) avesse scritto che i colori dello spettro sono divisi da intervalli «proportional to the seven Musical Tones or Intervals of the eight Sounds». Ciò dà l'opportunità a Voltaire di citare «un philosophe ingénieux», il gesuita Louis-Bertrand Castel, che aveva immaginato «un clavecin oculaire, qui doit faire paraître successivement des couleurs harmoniques, comme nos clavecins nous font entendre des sons». Castel non realizzò mai quel meraviglioso strumento: 
bisognava attendere l'èra dei computer perché qualcuno si cimentasse, con risultati controversi, nell'impresa; ma Voltaire pensava fosse opportuno ringraziare comunque pubblicamente un uomo «qui cherche à donner aux autres de nouveaux arts et de nouveaux plaisirs».

Un'alleanza tra bello e vero inseguì anche un amico italiano di Voltaire spesso ospite nel castello di Cirey, il veneziano Francesco Algarotti (1712-1764), autore di un Newtonianismo per le dame (1737) in forma di dialoghi redatto in chiara concorrenza con gli Élémens. Algarotti, tanto più giovane e tanto meno profondo del francese, aveva studiato seriamente soprattutto l'ottica, ripetendo tra i primi in Italia, presso l'Istituto delle scienze di Bologna, gli esperimenti con i prismi che confermavano la teoria corpuscolare newtoniana e la natura «settemplice» (parola non nuova ma di nuova applicazione) della luce. Algarotti aveva però avuto la debolezza di imitare, nello stile, gli Entretiens di Fontenelle, vecchi di cinquant'anni (e al sempre vegeto vegliardo il libro era infatti dedicato). Così, per esempio, nel libretto si poteva leggere, ad illustrazione della legge newtoniana del quadrato della distanza, una scipita galanteria di questo genere: «dopo otto giorni di assenza l'Amore è divenuto sessantaquattro volte minore di quello che fosse nel primo giorno» (dial. VI). Tuttavia il Newtonianismo di Algarotti (ristampato e tradotto in molte lingue, compreso l'inglese) conteneva pagine documentate e dimostrazioni solide. E ospitava, all'inizio, una polemica contro la filosofia aristotelica, malauguratamente accolta, lamentava, nella «monastica quiete» (gli scolastici venivano definiti «vecchi fanciulli» che combattevano con «bolle d'aria»); c'è anche un' eloquente difesa dei «martiri della Ragione», tra cui è menzione di «un uomo assai ardito, nomato Galileo, il czar Pietro il Grande della fisica». Ciò costò a 
quell'operetta, prediletta dalle dame ma non solo da loro, la condanna all'Indice (13 aprile 1739). L'Italia cattolica non era evidentemente ancora pronta per fare i conti con la propria storia, né disposta a riconoscere il valore universale delle verità scientifiche.

Quelle verità erano invece evidenti per i letterati di 'avanguardia' (chiamiamoli così) che popolavano la penisola? Non sempre. Giova fare il nome di un altro veneto, Antonio Conti (1677-1749), allora celebre per le sue tragedie e per i suoi versi (tra cui un'eccellente versione del Ricciolo rapito di Alexander Pope). Conti, curioso di scienza e di filosofia, aveva vissuto a lungo in Inghilterra, in Francia e in Germania, guadagnandosi la confidenza di Newton, oltreché di Leibniz. Sventuratamente ebbe l'idea di provare a riconciliare i due grandi matematici all'epoca della loro lite, insanabile, sulla priorità dell'invenzione del calcolo infinitesimale. Come spesso càpita ai pacieri, Conti finì per prender botte dall'uno e dall'altro, soprattutto dalla parte dell'inglese, notoriamente un po' misantropo. Fu in quell'occasione che Conti, piccato, si prese la libertà di scrivere, rivolto a Newton, quel che pensava del valore della «philosophie expérimentale» e delle «mathématiques» di cui un po' s'era dilettato:

J'aime beaucoup ces sortes d'études; mais elles ne m'inquièttent guère, et dans le fond je n'estime pas plus l'objet que la quadrille ou la chasse; tout cela revient au même, quand on l'examine sans passion; et d'ailleurs je suis persuadé que si on excepte quinze ou vingt problèmes utiles aux arts et aux usages de la société, tout le reste sera peut-être méprisé un jour comme certaines questions scholastiques, ou les question du 
vuide, des atomes, du tems, de la perfection de l'Universe, etc., que Mr. Newton méprise. (Lettre au sujet d'un petit écrit intitulé: Réponse aux observations sur la Chronologie de Mr. Newton, 1726)

Non bisogna prendere troppo sul serio l'amareggiato Conti: letterato raffinato ma non segnalato epistemologo. Eppure l'idea che trova eco nelle sue parole, cioè uno scetticismo profondo sul valore ultimo delle conoscenze scientifiche, era comune a molti dotti: e purtroppo forse lo è ancora. Diversamente pensava Voltaire, convinto d'esser nato in un'epoca dove «on commence à ouvrir les yeux et à jouir du plus bel appanage de l'humanité, l'usage de la raison». Per lui Newton era il gigante che meritava il famoso epitaffio di Pope, inciso a Westminster: «Nature, and Nature's Laws lay hid in Night. / God said: Let Newton be! And all was Light». Non che Voltaire credesse che quella luce potesse illuminare ogni angolo della Natura, della natura umana in particolare: «l'homme est une énigme obscure», leggiamo nel suo Poème sur la loi naturelle, 1752 (si pensi al tanto più tardo Faust goethiano: «der Mensch, die kleine Narrenwelt», l'uomo microcosmo di pazzia); né per Voltaire era molto più facile spiegare «cet instinct sûr et prompt qui sert les animaux», o il «germe impalpable» dell'erba «qui meurt pour renaître» (Darwin era ancora di là da venire). Bisognava ammetterlo: «sur ce vaste univers un grand voile est jeté»; però era ridicolo, e assurdo, pensare che nelle «profondeurs de cette obscurité» la luce della ragione potesse essere d'ostacolo: «Nous n'avons qu'un flambeau, gardons-nous de l'éteindre». È una domanda, anzi un'istanza drammatica che la letteratura fa: stavolta non soltanto inseguendo, ma raggiungendo la scienza. 